\title{
LIMONADA: A database dedicated to the simulation of biological membranes
}

\author{
Jean-Marc Crowet $^{1}$ ( $)$ | Sébastien Buchoux ${ }^{2}$ | Nicolas Belloy ${ }^{1}$ | \\ Catherine Sarazin $^{2}$ | Laurence Lins ${ }^{3}$ | Manuel Dauchez ${ }^{1}$
}

\author{
${ }^{1}$ Matrice Extracellulaire et Dynamique \\ Cellulaire (UMR CNRS 7369), Chaire MAgICS, \\ Université de Reims Champagne-Ardenne, \\ Reims, France \\ ${ }^{2}$ Unité de Génie Enzymatique et Cellulaire \\ (GEC-UMR7025 CNRS/UPJV/UTC), \\ Université de Picardie Jules Verne, Amiens, \\ France \\ ${ }^{3}$ Laboratoire de Biophysique Moléculaire aux \\ Interfaces, Université de Liège, Gembloux \\ Agro-Bio Tech, Gembloux, Belgium

\section{Correspondence} \\ Jean-Marc Crowet, Matrice Extracellulaire et \\ Dynamique Cellulaire (UMR CNRS 7369), \\ Chaire MAgICS, Université de Reims \\ Champagne-Ardenne, Reims 51687, France. \\ Email: jean-marc.crowet@univ-reims.fr \\ Funding information \\ Centre National de la Recherche Scientifique, \\ Grant/Award Number: SFR Condorcet- \\ FR3780; Fonds De La Recherche Scientifique - \\ FNRS, Grant/Award Numbers: CDR grant \\ J.0114.18, PDR grant T.1003.14; Université de \\ Reims Champagne-Ardenne, Grant/Award \\ Number: Chair of research MAgICS; Wallonie- \\ Bruxelles International, Grant/Award Number: \\ Excellence grant
}

\begin{abstract}
Cellular membranes are composed of a wide diversity of lipid species in varying proportions and these compositions are representative of the organism, cellular type and organelle to which they belong. Because models of these molecular systems simulated by MD steadily gain in size and complexity, they are increasingly representative of specific compositions and behaviors of biological membranes. Due to the number of lipid species involved, of force fields and topologies and because of the complexity of membrane objects that have been simulated, LIMONADA has been developed as an open database allowing to handle the various aspects of lipid membrane simulation. LIMONADA presents published membrane patches with their simulation files and the cellular membrane it models. Their compositions are then detailed based on the lipid identification from LIPID MAPS database plus the lipid topologies and the force field used. LIMONADA is freely accessible on the web at https://limonada. univ-reims.fr/.
\end{abstract}

\section{KEYWORDS}

database, force fields, lipid, model membrane, molecular dynamics

\section{INTRODUCTION}

Cellular membranes can be composed of hundreds of different lipid species distributed heterogeneously between and within bilayer leaflets and lipidomic studies have established that there could be over 100,000 different lipids. ${ }^{1-5}$ Resulting lipid compositions are organism, tissue, cell and organelle specific and several studies have yielded detailed membrane compositions of different cell and organelles types. ${ }^{6-8}$ Membrane composition can also evolve depending on environmental factors and during the cell cycle. ${ }^{9}$ Beside lipids, membranes are composed of proteins which represent up to $25 \%$ of the membrane surface at a protein/lipid ratio of 1/50-1/100 and those proteins depend on the lipid composition to function, can be recruited to specific domains and can present specific lipid interactions. ${ }^{10-14}$ With a modeling perspective, a large set of membrane models are then needed. ${ }^{15}$ During the last decade, the conjunction of a greater computing power and methodological developments has led to an ever-increasing size and complexity of the lipid systems simulated by molecular dynamics (MD) that enable the simulation of model membranes representative of cellular membranes. ${ }^{16-18}$ There is now tens of these model lipid membranes and they have been recently reviewed by Klauda in 2018 and Marrink et al. in 2019. ${ }^{15,19}$ For example, a model plasma membrane with 63 lipid types has been simulated by Ingolfsson et al. and an atomistic model of 100 million atom of an entire cell organelle, a photosynthetic chromatophore vesicle, has been simulated by Singharoy et al. ${ }^{20,21}$ Determination of complex lipid compositions is not trivial and simulating these membranes to equilibration can be quite computationally demanding while only being a prerequisite for further simulations. ${ }^{19}$ Indeed, model compositions are usually 
inferred from several experimental studies especially to include asymmetry information, which are usually missing from lipidomic studies and involve a careful choice of the lipid species to simulate. Regarding equilibration, while the time required for a membrane composed of a single type of lipid is in the order of $100 \mathrm{~ns}$, for a membrane with several lipid types this time is much longer. ${ }^{19}$ Model design also depends on the lipid parameters that have been developed for MD simulations. There is currently a wide diversity of lipids available, including fatty acyls, glycerolipids with PC, PE, PS, PI, PA, PG and glycosyl heads, ceramidebased lipids with PC (SM) and glycosyl heads, saccharolipids and sterols. Hydrocarbon chains, saturated or unsaturated, can be linked to the lipid core through ether or ester linkage and bear methyl or cyclopropane rings. ${ }^{22}$ The availability of parameters for those lipids depends on the force field and a recent review by Leonard et al. in 2019 details the history of lipid force field developments and evaluates the diversity of the lipids present for each force field. ${ }^{22}$ For instance, CHARMM $36,{ }^{23}$ MARTINI, ${ }^{24}$ and GROMOS ${ }^{25}$ force fields present the larger sets with several hundreds of lipid types readily available for CHARMM36 and MARTINI. ${ }^{22}$ Lipid parameters as well as simulated membranes can be obtained on different web sites or from the original authors. For example, CHARMM36 lipid parameters can be obtained from the MacKerell laboratory website (http://mackerell.umaryland.edu) or from the input generator of CHARMM-GUI (http://www.charmm-gui. org/) and lipid parameters and several membranes simulated with the MARTINI force field are available on the MARTINI website (http:// cgmartini.nl/). In 2010, the LIPIDBOOK database was created to gather lipid parameters (https://lipidbook.org/). ${ }^{26}$

The main goal of LIMONADA ("Lipid Membrane Open Network And Database") is to provide an online repository of membranes models in a simulation-ready fashion, including more complex compositions representative of biological membranes, along with the description of lipids, topologies and force fields mandatory for their MD simulations. Indeed, improvements in the knowledge of cellular membrane compositions due to experimental developments, notably from mass spectrometry, ${ }^{27}$ conjugated to modeling developments will eventually lead to a great number of representative model membrane compositions and there is actually no database dedicated to these objects. The number of lipid topologies available for simulation actually has also dramatically increased and can be replaced in the context of the lipid diversity encountered during biological studies, that is, a lipidomic database like LIPID MAPS (https://www.lipidmaps.org/). LIMONADA then also intends to use the LIPID MAPS developed classification and naming conventions to bridge lipid description to simulated membranes and the different force fields and softwares currently used for membrane simulations.

\section{2 | METHODS}

\subsection{Database and web development}

The LIMONADA website has been implemented through the Django web framework (https://www.djangoproject.com/) and the database format is sqlite3. LIMONADA is freely accessible on the web at https://limonada.univ-reims.fr/ and the source code, released under the GNU GPLv3, is available at https://github.com/LIMONADAMD. Registered users can add new entries in the database. By using Django, LIMONADA can also be used as a standalone application through a web browser.

LIMONADA is composed of four major parts dedicated to the description of lipids, topologies, force fields, and membranes and an overview schema of the database with the required information is presented on Figure 1. Each part presents records as tables and each record can be detailed through a personal page. Each part also presents a search section with various criteria and auto-completion is used wherever possible. The lipid classification of LIPID MAPS is used. ${ }^{28}$

No registration is required to access the data but users have to be registered to add records to the database. The only limitation is that each addition must be sustained by a reference paper to ensure that contributions are scientifically relevant. The primary sources of the files stored by LIMONADA are also provided for each entry. Once a user has added a new entry, he will become the curator of this entry. Curators do not need to be the creator of the data but the primary source should be given and the creators should be noticed. The curator of every entry is clearly specified on the website and their contact information are available. To promote discussion, a forum space is provided for each entry.

\section{2 | Lipid descriptions}

LIMONADA aims at gathering lipid parameters to allow the simulation of model membranes representative of biological membranes. To do so, we first need to have an idea of the lipids found in nature and an accurate description, notably from a structural point of view. Several lipid databases exist at the moment like SWISSLIPIDS (https://www. swisslipids.org/), ${ }^{29}$ LIPIDBANK (http://www.lipidbank.jp/), ${ }^{30}$ or LIPID MAPS (https://www.lipidmaps.org/). ${ }^{28}$ LIPID MAPS has more than 45,000 biologically relevant lipids, it is actively maintained and it is associated with classification and nomenclature systems which are internationally recognized and used by other projects and database, for example, by LION/Web. ${ }^{31}$ Moreover, a REST service allows to make HTTP requests on the database. Finally, it mostly describes lipids which correspond to a unique stereochemical structure. Since LIPID MAPS presents several advantages for the development of LIMONADA, we rely on this database for lipid description (Figure 1).

In LIMONADA, each lipid is linked to the corresponding LIPID MAPS entry and its standard denominations are used (Figure 1). When a lipid is not described in LIPID MAPS but has been used to simulate a membrane, a specific LIMONADA ID can be assigned automatically based on the lipid classification by using the same numeration principle that LIPID MAPS. In addition to this ID, a PubChem ID is also provided for each lipid to have access to a wider set of experimental information which are beyond the purpose of LIMONADA. Besides the usual lipid information, a short name is also assigned to each lipid. Indeed, most glycerophospholipid are defined by a fourcharacter name: The first and second digit for the sn1 and sn2 acyl 
FIGURE 1 LIMONADA database schema. Non mandatory fields are in italic, automatically generated fields are in gray, entries with dot lines cannot be filled directly by registered users

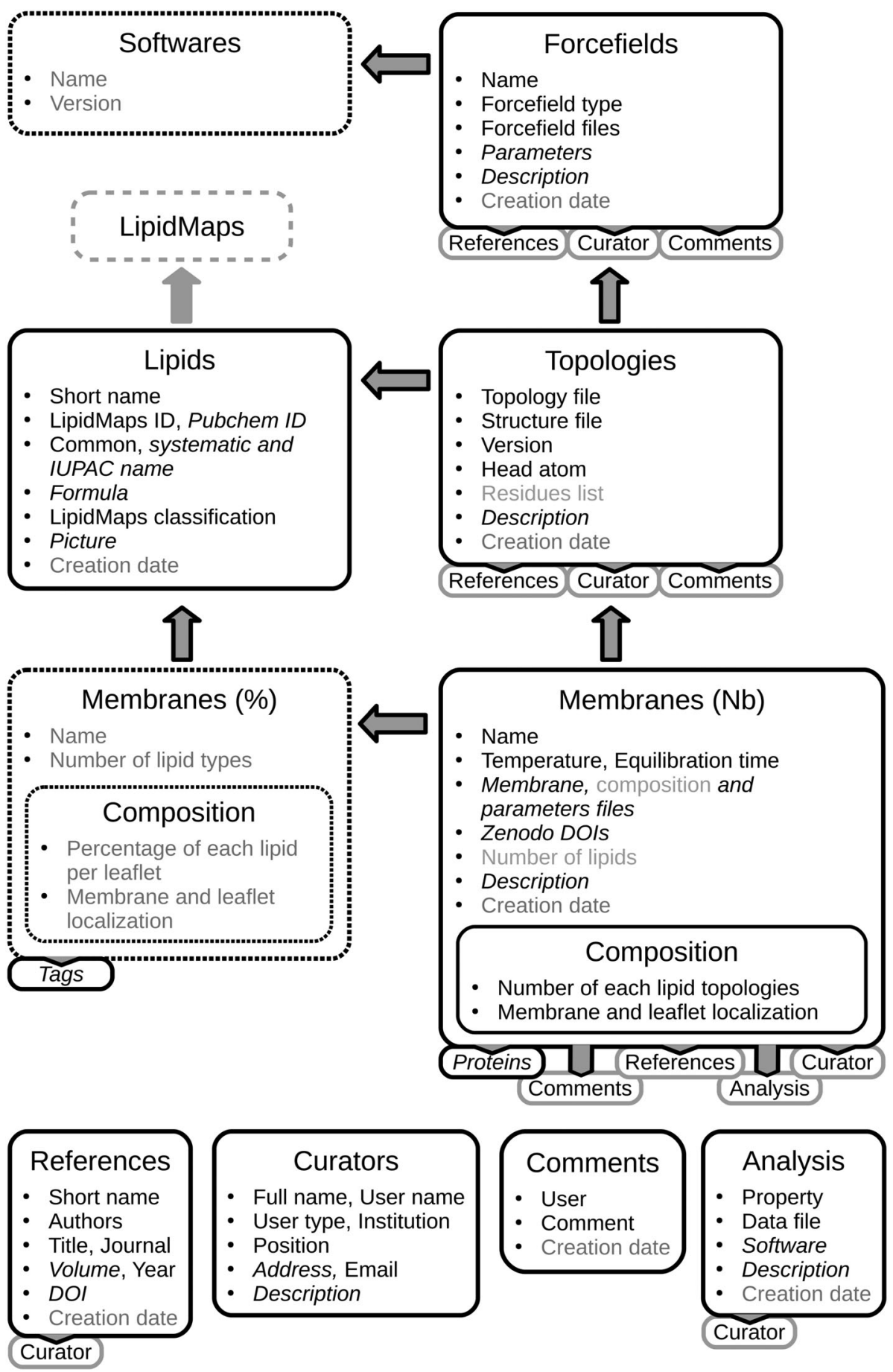

chains and the two last for the polar head group (i.e., POPC for palmitoyl-oleoyl-phosphatidyl-choline). As it is expected to be around 100,000 lipids, this name length has the advantage to be long enough to represent all these lipids and short enough to be used in the simulation files (i.e., compliant with e.g., pdb files).

During the addition of a lipid, almost all the information needed (Figure 1) are automatically gathered from the LIPID MAPS ID through an HTTP request and a figure is automatically drawn from the MOL file provided by using open babel (http://openbabel.org). ${ }^{32}$

Lipids can be searched by their lipid class or from their LIPID MAPS ID, common name and short name with the search box in the list banner (Figure S1). Due to the number of potentially matching lipids, this latter search is done through the use of an autocomplete field based on the substrings written by the user (i.e., if a user write "PC", all the lipids with "PC" in their LIPID MAPS ID, common name or short name will be displayed).

\section{\begin{tabular}{l|l}
2.3 & Topology and force field descriptions
\end{tabular}}

A lipid molecule can be described by several force fields and consequently by several MD topologies. LIMONADA distinguishes the 
description of the molecules from their force field-related topologies. When looking for a particular lipid composition to be simulated, one advantage of this choice is that users can start from the force field and look which lipids are available or vice versa. The four-character ID is used across all force field-dependent descriptions to unequivocally and coherently identify every lipid. This is quite useful given that name nomenclature can differ between force fields or change through time.

Force fields can be added for several versions of the three major MD softwares; CHARMM, GROMACS and AMBER, with the files necessary to run a simulation. Another file, containing the parameters (such as GROMACS mdp files), can also be provided along with the definition of the force field type (Atomistic, United atom or Coarse grained), references and additional descriptions. Softwares versions are discretized for two reasons. Some topologies depend on the version, in particular because the GROMACS definition of dihedral restraints has evolved between version 4.0 and 4.5 , and because the software version is also used in the description of membrane entries.

When a lipid and a force field are defined, a topology can be described by a topology file and a structure file. During the addition, topologies are checked against the force field by using the force field files and the structure provided with the software defined. For GROMACS, lipids with several residues can be added.

Once added, structure files can be visualized with the NGL viewer. ${ }^{33,34}$ The definition of a version name, a head atom and references are also mandatory. For one force field, several topologies entries can be used for a single lipid. Indeed, a lipid can be described differently in several references or the lipid can be represented in different ionization states. In LIMONADA, these topologies are distinguished by using different version names which usually correspond to the reference describing these parameters. If parameters have been modified compared to the published ones, it should be detailed in the description field or in a dedicated file. If these parameters are coming from other websites or databases, primary sources have also to be written in the description field. All the current data of LIMONADA have been added with the agreement of the creators or the web hosts of these parameters. The definition of an atom from the head is also mandatory because it will be used for the leaflet analysis of the membrane files described in the following section. Finally, references are mandatory to insure the scientific background of the data. References correspond to specific entries of the database and helps to keep tracks of sources of the data that have been added. During the addition of a reference, all the information needed can be automatically gathered from the DOI through an HTTP request on the DOI website (https://dx.doi.org/).

Like lipids, topologies can be searched according to the lipid class or from LIPID MAPS ID, common name and short name with the search box in the list banner (Figure S1). In addition, they can also be searched according to the softwares and force fields available in the database. To find a lipid through its original name, a search request can be done on the description field where the original name of the lipid is specified.

\section{4 | Membrane descriptions}

Model membranes are designed to be representative of specific biological membranes and can present different level of complexity (i.e., number of lipid types, presence of proteins or other molecules, geometry) depending on the experimental information, force field parameters and computational facilities at hand. Then for the same biological membrane, several model compositions can be designed and for a single model lipid composition, several molecular objects can be simulated. To take this into account, the model membrane composition (where the lipid content is expressed in percentage) is distinguished from the actual membrane object (where the number of lipid molecule is used). Several membrane objects can then have the same membrane composition and each composition is associated with several tags to identify which biological membrane it represents (i.e., "plasma membrane" or "saccharomyces cerevisiae"). Membrane compositions are created during the addition of a new membrane object if its composition does not exist. For membrane objects, the lipid composition of each membrane present in the structure file needs to be detailed with the number of each lipid per leaflet and with the topology used. To do so, once a force field has been chosen, the membrane file can be analyzed by FATSLiM to fill the composition form. ${ }^{35}$ FATSLiM is a fast tool which computes local membrane normal from lipid head atoms and uses a cut-off distance to extract the lipidic organization of simulated membranes independently of the bilayer morphology. The force field is used to display the topologies available for each lipid. After the FATSLiM analysis, lipids are organized by leaflet and reordered in the form and in the structure file according to the number of each lipid. If the same lipid has different topologies in the membrane, a fifth digit is added to the lipid name in the structure file to distinguish them from each other. As PDB files are limited to residue names of four digits, GRO and CRD files are created for GROMACSand CHARMMsoftwares, respectively. Membrane files are not mandatory but if present, the lipid composition detailed in the form must correspond to the lipid order in the structure file to be validated. On membrane addition, other simulation-related information have also to be indicated, like temperature and equilibration time, along with references, additional files (i.e., parameters files), Zenodo DOls and descriptions (i.e., primary sources of files and potential modifications made to the files). Besides lipids, proteins and other molecules can also be simulated with membranes. For proteins, a specific database entry has been created while other molecules can be described in the description field. Once a membrane has been added, the structure file can be visualized with a NGL viewer and Figure S2 shows an example of a description page for a membrane. From this page, the files required to simulate this membrane, especially the lipid topologies, can be downloaded. Membrane tags as well as protein information are independent database entries that can be created during membrane addition and that can be searched for in the search banner. Membranes can be also searched based on the lipids used and according to the softwares and force fields available in the database. Physical properties of the membranes, such as area per lipid, 
membrane thickness and order parameters, can be associated to each membrane record and visualized.

\section{3 | DISCUSSION}

Cellular membranes are complex molecular objects which present a wide diversity of lipid species. Recent reviews have shown that lipid parameters have been actively developed for several force fields during the last decade and that they have been used to tackle the simulation of increasingly realistic cellular membranes. ${ }^{15,19}$ In this context, LIMONADA intends to reflect the diversity of the information available for membrane simulations and puts it in the lipidomic context with an open approach. To the knowledge of the authors, a database which describes model membranes that can be downloaded and seamlessly used in MD simulations does not exist.

A few databases provide parameters for MD simulations of lipids and/or membranes. MemProtMD (http://memprotmd.bioch.ox.ac. $\mathrm{uk} /$ ) is dedicated to membrane proteins and provides MD-ready files of proteins embedded into a DPPC lipid bilayer. CHARMM-GUI (http://www.charmm-gui.org/) provides a great interface to build lipid bilayers from scratch and simulate these systems with CHARMM36 or MARTINI force field mainly. It is thus not a database per se but lipid systems made using CHARMM-GUI can be imported into LIMONADA. LIPIDBOOK (https://lipidbook.bioch.ox.ac.uk/) is a public repository for lipid force field parameters focusing on lipid topologies. From this point of view, the code and database availability plus several aspects of its integrated approach, LIMONADA hence represents a step forward compared to the LIPIDBOOK database. ${ }^{26} \mathrm{NMRLipids}$ (https://zenodo.org/communities/nmrlipids/) in an open scientific collaboration comparing lipid parameters from several force fields with experimental data to understand the atomistic resolution structures of lipid bilayers. ${ }^{36}$ It is not a database per se either but simulation data are deposited on a Zenodo community space. Although somehow different, resources like SWISSLIPID and LIPIDBUILDER are also providing useful information on lipid biophysical parameters or to build membranes, respectively. To credit these sources, a link page has been created on the LIMONADA website.

The process of adding records has been thought to be as simple as possible, with fill helper for lipids from LIPID MAPS ID, for references from DOI and for membrane compositions from membrane files and force field selected. Tests were also implemented to check the consistency of the entries, short simulations being carried when a topology is added and by comparing the membrane composition with the membrane file. The file formats to upload lipid and membranes structures is currently limited to PDB and GRO files but other files formats, notably CRD, are will be added in future release. Lastly, curators are clearly identified, can be easily contacted and each entry can be discussed through a forum section. This enables a control of the deposited data from experts of the field. These features in addition to the openness of the project (availability of the database and of the code on github) tend to promote the use and the deposit of new files by the users. For instance, the project can be used and developed in house.

Usually, when authors make available a structure file of the membrane simulated, it corresponds to the last frame of the simulation, with sometimes several intermediary frames or MD trajectories. Due to the size of these files, the LIMONADA database does not intend to host MD trajectories. Specific hosting services like Zenodo can be used for this purpose.

A database, such as LIMONADA, is very useful as MD simulations tackle more and more complex and accurate description of biological membranes. Hence, when searching for a specific model membrane or designing a new one, LIMONADA database would reduce the need to construct and equilibrate complex lipid membranes if already existing. Determination of complex lipid compositions is not trivial and their equilibration can be quite computationally demanding while only being a prerequisite for further simulations. Besides advantages and limitations of each force field, the number of lipid topologies is also strongly force field dependent and LIMONADA can easily be used to choose a force field from a selected set of lipids or inversely see which lipids are available for a selected force field.

At a more lipidomic point of view, LIMONADA has also several advantages. First, it enables to highlight missing data relative to biologically relevant lipids. For example, some main plant lipids like glycosylated and acetylglycosylated sterols, which may represent a significant amount of the plasma membrane of tobacco, lack parametrization. ${ }^{37}$ Second, it enables to have a coherent lipid naming and classification across different force fields, in particular lipid short names which are often different from one force field to another.

\section{4 | CONCLUSIONS}

The number and the complexity of lipid compositions are believed to increase dramatically during the coming years. Filling a database is a demanding process but the project has been thought to enable researchers working in the field to add their own contributions if there are willing to. Indeed, a lot of published lipid topologies have been parameterized and published as well as multi components membranes but they are only available upon request to the authors. This situation could be due to the lack of a dedicated and user friendly platform (except LIPIDBOOK). LIMONADA could host these data belonging to the gray part of science and then facilitating the simulation of representative model membranes.

\section{ACKNOWLEDGMENTS}

This work was partly funded by FR3780 CNRS-SFR Condorcet, by the chair of research MAgICS and the Wallonie-Bruxelles International (WBI) administration through an excellence grant. Laurence Lins is senior research associate for the Belgian Fund for Scientific Research (FRS-FNRS) and thanks for financial support (FNRS CDR grant J.0114.18 and PDR grant T.1003.14). This work had the support of both the supercenter of calculation ROMEO and Multiscale Molecular Modeling Platforms of the University of Reims Champagne-Ardenne. 


\section{ORCID}

Jean-Marc Crowet (D) https://orcid.org/0000-0002-9252-2396

\section{REFERENCES}

[1] K. Jacobson, O. G. Mouritsen, R. G. W. Anderson, Nat. Cell Biol. 2007, $9,7$.

[2] G. van Meer, D. R. Voelker, G. W. Feigenson, Nat. Rev. Mol. Cell Biol. 2008, 9, 112.

[3] T. Kobayashi, A. K. Menon, Curr. Biol. 2018, 28, R386.

[4] H. I. Ingólfsson, T. S. Carpenter, H. Bhatia, P.-T. Bremer, S. J. Marrink, F. C. Lightstone, Biophys. J. 2017, 113, 2271.

[5] J.-L. Cacas, C. Buré, K. Grosjean, P. Gerbeau-Pissot, J. Lherminier, Y. Rombouts, E. Maes, C. Bossard, J. Gronnier, F. Furt, L. Fouillen, V. Germain, E. Bayer, S. Cluzet, F. Robert, J.-M. Schmitter, M. Deleu, L. Lins, F. Simon-Plas, S. Mongrand, Plant Physiol. 2016, 170, 367.

[6] A. Y. Andreyev, E. Fahy, Z. Guan, S. Kelly, X. Li, J. G. McDonald, S. Milne, D. Myers, H. Park, A. Ryan, B. M. Thompson, E. Wang, Y. Zhao, H. A. Brown, A. H. Merrill, C. R. H. Raetz, D. W. Russell, S. Subramaniam, E. A. Dennis, J. Lipid Res. 2010, 51, 2785.

[7] J. L. Sampaio, M. J. Gerl, C. Klose, C. S. Ejsing, H. Beug, K. Simons, A. Shevchenko, Proc. Natl. Acad. Sci. U. S. A. 2011, 108, 1903.

[8] C. S. Ejsing, J. L. Sampaio, V. Surendranath, E. Duchoslav, K. Ekroos, R. W. Klemm, K. Simons, A. Shevchenko, Proc. Natl. Acad. Sci. U. S. A. 2009, 106, 2136.

[9] T. Harayama, H. Riezman, Nat. Rev. Mol. Cell Biol. 2018, 19, 281.

[10] A. D. Dupuy, D. M. Engelman, Proc. Natl. Acad. Sci. U. S. A. 2008, 105, 2848.

[11] A. L. Duncan, T. Reddy, H. Koldsø, J. Hélie, P. W. Fowler, M. Chavent, M. S. P. Sansom, Sci. Rep. 2017, 7, 16647.

[12] T. Ryan, J. Myers, D. Holowka, B. Baird, W. Webb, Science 1988, 239, 61.

[13] M. Javanainen, H. Hammaren, L. Monticelli, J.-H. Jeon, M. S. Miettinen, H. Martinez-Seara, R. Metzler, I. Vattulainen, Faraday Discuss. 2013, 161, 397.

[14] R. Friedman, S. Khalid, C. Aponte-Santamaría, E. Arutyunova, M. Becker, K. J. Boyd, M. Christensen, J. T. S. Coimbra, S. Concilio, C. Daday, F. J. van Eerden, P. A. Fernandes, F. Gräter, D. Hakobyan, A. Heuer, K. Karathanou, F. Keller, M. J. Lemieux, S. J. Marrink, E. R. May, A. Mazumdar, R. Naftalin, M. Pickholz, S. Piotto, P. Pohl, P. Quinn, M. J. Ramos, B. Schiøtt, D. Sengupta, L. Sessa, S. Vanni, T. Zeppelin, V. Zoni, A.-N. Bondar, C. Domene, J. Membr. Biol. 2018, 251, 609.

[15] S. J. Marrink, V. Corradi, P. C. T. Souza, H. I. Ingólfsson, D. P. Tieleman, M. S. P. Sansom, Chem. Rev. 2019, 119, 6184.

[16] F. J. van Eerden, D. H. de Jong, A. H. de Vries, T. A. Wassenaar, S. J. Marrink, Biochim. Biophys. Acta, Biomembr. 2015, 1848, 1319.

[17] N. Flinner, E. Schleiff, PLoS One 2015, 10, e0133999.

[18] H. Koldsø, D. Shorthouse, J. Hélie, M. S. P. Sansom, PLoS Comput. Biol. 2014, 10, e1003911.

[19] J. B. Klauda, J. Chem. Phys. 2018, 149, 220901.

[20] H. I. Ingólfsson, M. N. Melo, F. J. van Eerden, C. Arnarez, C. A. Lopez, T. A. Wassenaar, X. Periole, A. H. de Vries, D. P. Tieleman, S. J. Marrink, J. Am. Chem. Soc. 2014, 136, 14554.
[21] A. Singharoy, C. Maffeo, K. H. Delgado-Magnero, D. J. K. Swainsbury, M. Sener, U. Kleinekathöfer, J. W. Vant, J. Nguyen, A. Hitchcock, B. Isralewitz, I. Teo, D. E. Chandler, J. E. Stone, J. C. Phillips, T. V. Pogorelov, M. I. Mallus, C. Chipot, Z. Luthey-Schulten, D. P. Tieleman, C. N. Hunter, E. Tajkhorshid, A. Aksimentiev, K. Schulten, Cell 2019, 179, 1098.

[22] A. N. Leonard, E. Wang, V. Monje-Galvan, J. B. Klauda, Chem. Rev. 2019, 119, 6227.

[23] J. B. Klauda, R. M. Venable, J. A. Freites, J. W. O'Connor, D. J. Tobias, C. Mondragon-Ramirez, I. Vorobyov, A. D. MacKerell, R. W. Pastor, J. Phys. Chem. B 2010, 114, 7830.

[24] S. J. Marrink, A. H. de Vries, A. E. Mark, J. Phys. Chem. B 2004, 108,750 .

[25] C. Oostenbrink, A. Villa, A. E. Mark, W. F. van Gunsteren, J. Comput. Chem. 2004, 25, 1656.

[26] J. Domański, P. J. Stansfeld, M. S. P. Sansom, O. Beckstein, J. Membr. Biol. 2010, 236, 255.

[27] L. Li, J. Han, Z. Wang, J. Liu, J. Wei, S. Xiong, Z. Zhao, Int. J. Mol. Sci. 2014, 15, 10492.

[28] E. Fahy, S. Subramaniam, R. C. Murphy, M. Nishijima, C. R. H. Raetz, T. Shimizu, F. Spener, G. van Meer, M. J. O. Wakelam, E. A. Dennis, J. Lipid Res. 2009, 50 Suppl, S9.

[29] L. Aimo, R. Liechti, N. Hyka-Nouspikel, A. Niknejad, A. Gleizes, L. Götz, D. Kuznetsov, F. P. A. David, F. G. van der Goot, H. Riezman, L. Bougueleret, I. Xenarios, A. Bridge, Bioinformatics 2015, 31, 2860.

[30] K. Watanabe, E. Yasugi, M. Oshima, Trends Glycosci. Glycotechnol. 2000, 12, 175.

[31] M. R. Molenaar, A. Jeucken, T. A. Wassenaar, C. H. A. van de Lest, J. F. Brouwers, J. B. Helms, Gigascience 2019, 8, giz061. https://doi. org/10.1093/gigascience/giz061.

[32] N. M. O'Boyle, M. Banck, C. A. James, C. Morley, T. Vandermeersch, G. R. Hutchison, Aust. J. Chem. 2011, 3, 33.

[33] A. S. Rose, P. W. Hildebrand, Nucleic Acids Res. 2015, 43, W576.

[34] A. S. Rose, A. R. Bradley, Y. Valasatava, J. M. Duarte, A. Prlić, P. W. Rose, Bioinformatics 2018, 34, 3755.

[35] S. Buchoux, Bioinformatics 2017, 33, 133.

[36] O. H. S. Ollila, G. Pabst, Biochim. Biophys. Acta, Biomembr. 2016, 1858, 2512.

[37] F. Furt, F. Simon-Plas, S. Mongrand, in The Plant Plasma Membrane, Vol. 19 (Eds: A. Murphy, B. Schulz, W. Peer), Springer, Berlin, Heidelberg 2011, p. 3.

\section{SUPPORTING INFORMATION}

Additional supporting information may be found online in the Supporting Information section at the end of this article.

How to cite this article: Crowet J-M, Buchoux S, Belloy N, Sarazin C, Lins L, Dauchez M. LIMONADA: A database dedicated to the simulation of biological membranes. J Comput Chem. 2021;1-6. https://doi.org/10.1002/jcc.26511 